\title{
BRD8 is a potential chemosensitizing target for spindle poisons in colorectal cancer therapy
}

\author{
HIROSHI Y. YAMADA and CHINTHALAPALLY V. RAO \\ Department of Medicine, Hematology/Oncology Section, University of Oklahoma \\ Health Sciences Center (OUHSC), OK 73104, USA
}

Received April 29, 2009; Accepted June 19, 2009

DOI: 10.3892/ijo_00000425

\begin{abstract}
Survival rate of metastatic colorectal cancers is less than $5 \%$. A major reason is that those cancers respond poorly to chemotherapy drugs. However, factors contributing to chemoresistance in colorectal cancers are barely known, thus isolation of factors involved is the critical first step for mechanistic understanding and therapy improvement. With expression cloning, we isolated human BRD8 (bromodomain 8 ) as an influential factor for spindle poison sensitivity. BRD8 is an accessory subunit of human NuA4-HAT (histone acetyl transferase) complex (also known as TRRAP/TIP60 complex), but its role in cancer and drug resistance is unknown. Here, we report that BRD8 is involved in cellular survival and in sensitivity to spindle poisons and proteasome inhibitor in aggressive colorectal cancers. BRD8 protein expression level is several-fold higher in human metastatic colorectal cancer cell lines (DLD-1, HCA-7 and HCT-116) than in other cell lines tested. Normal-appearing rat colonic mucosa and azoxymethane (AOM)-induced colorectal adenocarcinoma tissue expressed a barely detectable amount of BRD8 protein, but aggressive colon tumors induced with AOM and dextran sodium sulfate expressed BRD8 at a significantly higher level, suggesting that BRD8 expression is associated with tumor progression toward advanced stages and may aid to gain growth advantage. Supporting the notion, siRNA-mediated knockdown of BRD8 induced cell death or growth delay in colorectal cancer cell lines, and surviving BRD8-knockdown cells were particularly sensitive to spindle poisons and a proteasome inhibitor MG132. Conversely, at least one isoform of BRD8 gave growth advantage and resistance to taxol when stably overexpressed in HeLa cells.
\end{abstract}

Correspondence to: Dr Hiroshi Y. Yamada, OUHSC, BRC1207, 975 NE 10th St., Oklahoma City, OK 73104, USA

E-mail: hiroshi-yamada@ouhsc.edu

Abbreviations: AOM, azoxymethane; BRD8, bromodomain 8; DSS, dextran sodium sulfate; HAT, histone acetyl transferase

Key words: taxol, spindle, mitosis, microtubule, BRD8, TIP60NuA4 HAT complex, colorectal cancer
Targeting BRD8 would improve therapy outcome against aggressive/metastatic colorectal cancers.

\section{Introduction}

Chemotherapy is a frontline tool in the treatment of a variety of cancers. One important class of chemotherapy drugs are spindle poisons or microtubule inhibitors [e.g., vinka alkaloids (vinorelbine, vinblastine), taxanes and taxoteres (taxol/ paclitaxel, docetaxel)] which are widely used in the treatment of several types of cancers. These drugs directly bind to tubulin, a component of microtubules and inhibit the function of microtubules (1,2 and 3). When the cells enter mitosis, the drugs inhibit mitosis-specific bundle of microtubules, the mitotic spindle. In the presence of a spindle defect, mitotic spindle checkpoint blocks mitotic metaphase-anaphase transition through inhibition of a ubiquitin ligase complex called anaphase promoting complex/cyclosome $(4,5)$. Thus, spindle poisons cause spindle checkpoint-mediated mitotic arrest/delay at the cellular level, and cell death or senescence eventually follows $(6,7)$, but with considerable variations in the pheno-type and in the degree of response $(8,9)$. The spindle poison-mediated cell death/senescence signaling pathway is currently under extensive investigation. Recent shRNA library-based screening suggested that sensitivity to spindle poison is influenced by a number of genes $(10,11)$, and how these gene functions affect spindle poison sensitivity is a subject that awaits further investigation.

Although spindle poisons are effective for the treatment of cancers of breast, lung, and ovary, the drugs show only marginal therapeutic benefits on colorectal cancers, especially in metastatic stage; thus, spindle poisons are not actively used for colorectal cancer treatment (12). Another class of promising chemotherapy drug proteasome inhibitors (e.g., velcade/bortezomib/PS341) is effective for treatment of myeloma and lymphoma, but failed to inhibit metastatic colorectal tumors in a single agent phase II clinical trial (13). Given less than 5\% survival rate of metastatic colorectal cancer patients, improvement strategies for effective therapy is imperative. Understanding the molecular pathways involved in resistance against spindle poison-mediated therapy will allow development of novel targeted strategies that will aid to sensitize non-responsive cancer cells such as metastatic colorectal cancers to respond to therapy.

To discover factors that influence spindle poison sensitivity, we previously developed a HeLa cell-based cDNA 
expression cloning method (14). This method yielded several cDNA fragments whose expression sensitized HeLa cells to spindle poisons. Here we report that BRD8, one of the candidates encoded by the cDNA fragments, is a factor overexpressed in aggressive colorectal cancers and rendering growth advantage and spindle poison resistance.

\section{Materials and methods}

Cell culture. The cell lines were obtained from the American Type Culture Collection (ATCC). We followed the ATCC guidelines for culture conditions. HCT-116, HT-29, SkBr-3, and HCA-7 were maintained in McCoys 5A with $1.5 \mathrm{mM}$ L-glutamate (Cellgro, 10-050-CV) and 10\% FBS (Sigma, F4135). Caco-2 and HeLa were maintained in MEM with $2 \mathrm{mM}$ L-glutamate, $0.1 \mathrm{mM}$ NEAA, $1 \mathrm{mM}$ sodium pyruvate (Cellgro, 10-010-CV) and 10\% FBS. DLD-1, PANC-1 and BxPC-3 were maintained in RPMI-1640 (Cellgro, 10-040$\mathrm{CV}$ ) with $10 \%$ FBS. SW-408 were maintained in DMEM with $4.5 \mathrm{~g} / 1$ glucose, L-glutamate and sodium pyruvate (Cellgro, 10-013-CV) with 10\% FBS. Penicillin-streptomycin (Cellgro, 30-002-CI) was added to all media (1\% v/v). Cells were kept in $37^{\circ} \mathrm{C}$ in $5 \% \mathrm{CO}_{2}$.

Rat colonic mucosa/tumor samples. Colon carcinogenesis was initiated in 8-week-old male F344 rats with azoxymethane (AOM) $(15 \mathrm{mg} / \mathrm{kg}$ body weight, once/week administered subcutaneously for two weeks), and tumor and control samples were harvested 12 months later. To obtain aggressively growing colorectal tumors, male F344 rats were treated with AOM as above, followed by $1 \%$ dextran sodium sulfate (DSS) (molecular weight 50,000) treatment in drinking water three days/week for two weeks. Once the AOM/DSS treatment was completed, the animals were maintained for four months, and then they were sacrificed and normal-appearing colonic mucosal tissue and tumors were harvested. Small portions of each rat colon tumor were fixed in $10 \%$ buffered formalin for histopathology analysis.

Immunoblotting. Cultured cells were extracted in extraction buffer $[1 \%(\mathrm{w} / \mathrm{w}) \mathrm{NP} 40,1 \%(\mathrm{w} / \mathrm{v})$ sodium deoxycholate, $0.1 \%$ SDS, $1 \%$ Triton $\mathrm{X}-100,10 \mathrm{mmol} / \mathrm{l}$ sodium phosphate (pH 7.2), 2 mmol/1 EDTA, $150 \mathrm{mmol} / 1 \mathrm{NaCl}, 50 \mathrm{mmol} / 1$ $\mathrm{NaF}$, and $0.2 \mathrm{mmol} / \mathrm{l}$ sodium vanadate] supplemented with $10 \mu \mathrm{mol} / \mathrm{l} \mathrm{MG} 132,5 \mu \mathrm{g} / \mathrm{ml}$ protease inhibitor cocktail (Sigma, P8340) and $0.5 \%(\mathrm{v} / \mathrm{v})$ phosphatase inhibitor cocktail 1 (Sigma, P2850), and boiled with SDS loading buffer for 7 minutes. Protein concentration was measured with the BCA protein assay kit (BioRad) and protein amounts loaded on the gels were equalized (15 $\mu \mathrm{g} /$ lane). Tumor tissue sample were homogenized with extraction buffer. We used the following antibodies for this study: anti-BRD8 (Bethyl laboratory A300-219A, Montgomery, TX), anti-ß-actin (Abcam, Cambridge, MA), anti-histone $\mathrm{H} 3$ and phosphorylatedhistone H3 S10 (Cell Signaling Technology, Danvers, MA).

Fluorescence-activated cell sorting (FACS) analysis for cell cycle profiling. FACS samples were harvested with trypsinization at indicated time, fixed with $-20^{\circ} \mathrm{C} 90 \%$ ethanol/10\% PBS and stored for at least $6 \mathrm{~h}$ until use. The cells were rehydrated with PBS, treated with ribonuclease A $(100 \mu \mathrm{g} / \mathrm{ml})$ and propidium iodide $(20 \mu \mathrm{g} / \mathrm{ml})$ in PBS with $50 \mathrm{mM}$ sodium citrate for $18 \mathrm{~h}$ at room temperature and subjected to FACS analysis after nylon mesh filtration to remove cell aggregates. FACS analysis was performed using FACS-calibur flowcytometer with the aid of OUHSC flow cytometry laboratory. Analysis of data was performed with Modfit software. FACS experiments were repeated at least twice.

siRNA transfection. SiRNA for BRD8 was purchased from Invitrogen (stealth siRNA). Six siRNAs for BRD8 were tested for knockdown, and we present the results from a mixture of three siRNAs $(01,03$ and 05$)$ in this report. The target sequences were: 01, CCACAAGUAUGGAGCCACCAGA ACU; 03, CCCUGAAAGCAUGUUGUCUCCAUCA; and 05, GGCCUAUGGAUUUGUCAACUAUUAA. Negative controls used had no siRNA with transfection reagents. GFP-specific siRNA with transfection reagents was also used for negative control. Transfection was performed with oligofectamine reagent (Invitrogen) following manufacturer's instructions. Briefly, for transfection of one well in a 6-well plate, $10 \mu \mathrm{l}$ of $20 \mu \mathrm{M}$ siRNA duplex and $3 \mu \mathrm{l}$ of oligofectamine were mixed in $200 \mu \mathrm{l}$ serum-free medium then added to $800 \mu 1$ of cells in serum-containing medium.

Viability/colony formation assay. Colony formation assays were performed and quantified as described previously (15). For cell staining in microscopic assay, acrydine orange $(100 \mu \mathrm{g} / \mathrm{ml})$ and ethidium bromide $(100 \mu \mathrm{g} / \mathrm{ml})$ in PBS was directly added to culture medium and cells were observed 5 min later under a microscope (16). Samples were observed in an Olympus IX71 inverted microscope connected to light sources TH4-100 and X-Cite 120 (EXFO). Images were processed with DP manager software (Olympus).

Generation of BRD8-overexpressing cell lines. We transfected HeLa cells with $1 \mu \mathrm{g}$ of BRD8 expression constructs, pCAGGS-BRD8-1-HA or pCAGGS-BRD8-2-HA (a generous gift from Dr Furukawa, University of Tokyo, Japan), along with $0.05 \mu \mathrm{g}$ pHygEGFP (Clontech) for selection marker. pCAGGS-BRD8-1-HA expresses shorter isoform 1, and pCAGGS-BRD8-2-HA expresses longer isoform 2. After selection in $100 \mu \mathrm{g} / \mathrm{ml}$ hygromycin-containing medium for three weeks, surviving colonies were pooled and subjected to immunoblotting to confirm BRD8 overexpression.

\section{Results}

Isolation of BRD8 as a candidate for spindle poison sensitivity influencing factor. We previously performed a HeLa cellbased cDNA-expression screening for factors that affect spindle poison sensitivity (14). A cDNA fragment of BRD8 gene was isolated from the screening along with several other cDNA fragments including TRIP1, a dual function protein that functions as a thyroid hormone receptor interacting protein and a component of $19 \mathrm{~S}$ proteasome (15). Human BRD8 gene locates on chromosome 5q31.2, and is widely conserved among higher eukaryotes. BRD8 has at least two major isoforms, isoform 1 (951AA, calculated weight 102.8 $\mathrm{kDa}$ ) and isoform 2 (1235AA, $135.4 \mathrm{kDa}$ ) (Fig. 1A). These isoforms have shared $\mathrm{N}$-terminus with variable $\mathrm{C}$-terminus 
Brd8/ Thyroid Hormone Receptor coactivator isoform 1

951AA

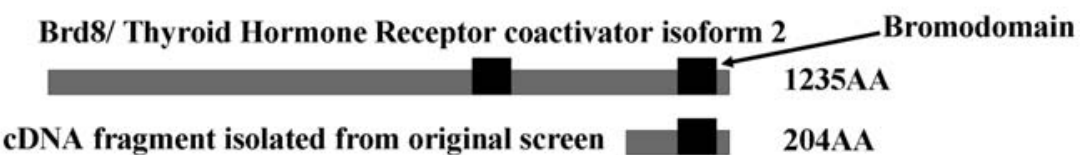

cDNA fragment isolated from original screen $\quad$ 204AA

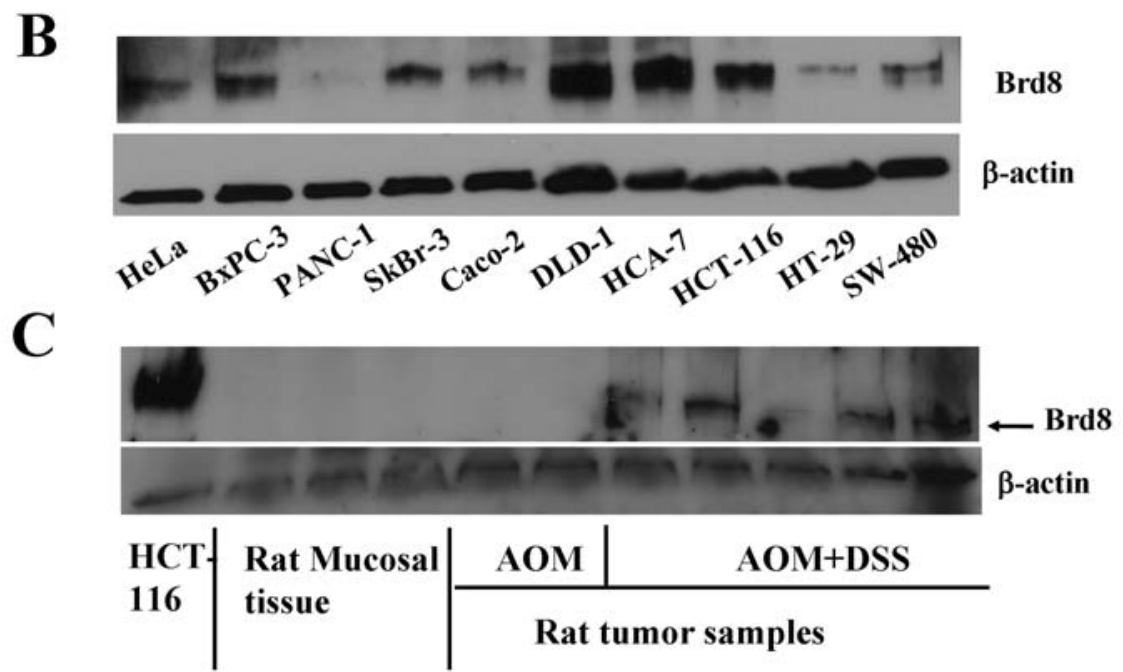

Figure 1. BRD8 protein expression is elevated in advanced colorectal tumors. (A) Protein structures of BRD8 isoforms 1 and 2 , and BRD8 C terminus region encoded by the cDNA fragment from the original screen. Black box represents bromodomain, an acetylated lysine-binding domain. (B) Immunoblots of BRD8 and B-actin (loading control) in a set of cultured cells. (C) Immunoblots of BRD8 and B-actin (loading control) in HCT-116 (lane 1, control), rat colorectal mucosal tissue (lanes 2-4), rat AOM-induced tumor (lanes 5 and 6), and rat AOM and DSS-induced advanced tumors (lanes 7-11).

due to differential splicing. Both isoforms are an accessory subunit of NuA4 HAT complex (also known as TRRAP/ TIP60 complex), which is involved in modification of chromatin structure and regulation of transcription, and can be purified as a part of the complex by biochemical means $(17,18)$. The cDNA expression cloning isolated a part of BRD8 isoform 2, 204 amino acids long $\mathrm{C}$ terminus region containing bromodomain. Given the small size of isolated cDNA fragment, we suspected that the expression of the $\mathrm{C}$ terminus fragment may work as dominant negative and inhibitory to BRD8 function. Since effective in vitro or in vivo assay to correlate BRD8 function to spindle poison sensitivity was not established, instead of testing inhibitory effect of expressing small cDNA fragment in vitro, we proceeded to characterize BRD8 and tested whether inhibition of BRD8 via siRNA sensitizes cells to spindle poison to validate the potential as a therapeutic target.

BRD8 protein expression is elevated in metastatic and highly proliferating colorectal cancer cell lines. To investigate BRD8 function and the effect of BRD8 inhibition, we first probed BRD8 protein expression in a set of human cultured cells with anti-human BRD8 antibody, which recognizes both isoforms. As shown in Fig. 1B, BRD8 protein expression level in exponentially growing cells was variable among cell lines, and was elevated several-fold in metastatic colorectal cancer cell lines DLD-1, HCA-7, and HCT-116 compared to HeLa (cervical cancer), SkBr-3 (breast cancer), PANC-1 and BxPC-3 (pancreas cancer), or the slow proliferating colorectal tumor cell line $(\mathrm{Caco}-2)$ that retains some characteristics of primary colonocytes.
BRD8 protein expression is elevated in aggressively growing rat colorectal cancer. With the observation of BRD8 amount increase in aggressive human colorectal tumor-origin cell lines, we questioned whether the BRD8 increase can be observed in tumors. We tested BRD8 protein expression in rat colorectal tumor samples available from our chemoprevention research (Fig. 1C). Normal-appearing rat colonic mucosa and azoxymethane (AOM)-induced colorectal adenocarcinoma tissue expressed barely detectable amount of BRD8 protein. However, aggressively growing tumors derived from simultaneous treatment with AOM and DSS expressed BRD8 at a significantly higher level. Simultaneous treatment with AOM and DSS is known to produce aggressive adenocarcinomas, which develop larger in a shorter time period than colon adenocarcinomas with single AOM treatment (19). These results suggest that higher BRD8 protein amount is associated with advanced colorectal tumors.

siRNA-mediated BRD8 knockdown is inhibitory to growth of cultured cells to a varying degree. To investigate the effect of the reduction of BRD8 protein amount, we used siRNA transfection. We first tested a set of BRD8 siRNA constructs for knockdown efficiency, along with two negative controls (empty transfection and GFP-targeting siRNA transfection) in human colorectal cancer cell line HCT-116 (Fig. 2A), in which BRD8 expression is higher and assessment of knockdown efficiency is feasible (Fig. 1A). Among six BRD8specific constructs $(01,03,05, \mathrm{~s} 1, \mathrm{~s} 2$ and $\mathrm{s} 3)$, one construct (01) showed over $95 \%$ inhibition compared with controls (- and GFP), two (03 and 05) showed 90\% inhibition, and three (s1, s2 and s3) showed 60-80\% inhibition $48 \mathrm{~h}$ after 
A

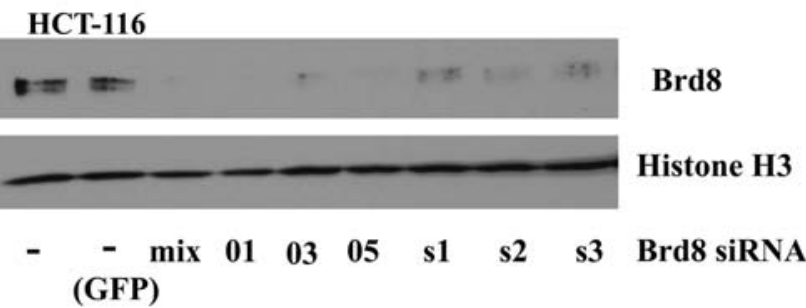

B

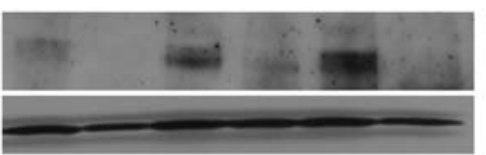

Brd8

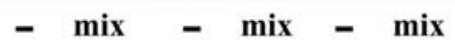

Histone $\mathrm{H3}$

HCT-116 HCA-7 DLD-1

Brd8 siRNA(mix)

C

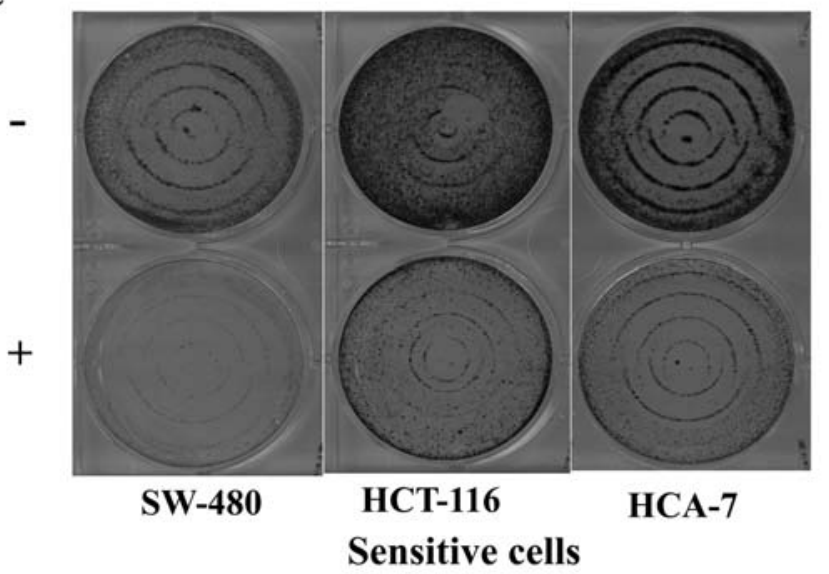

Cell lines

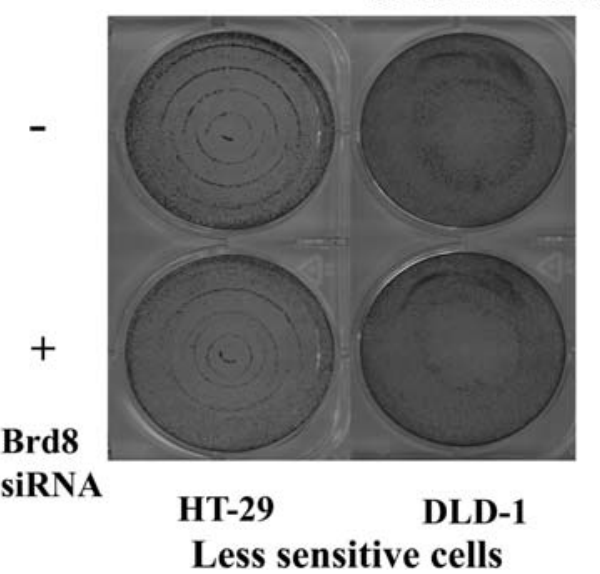

D

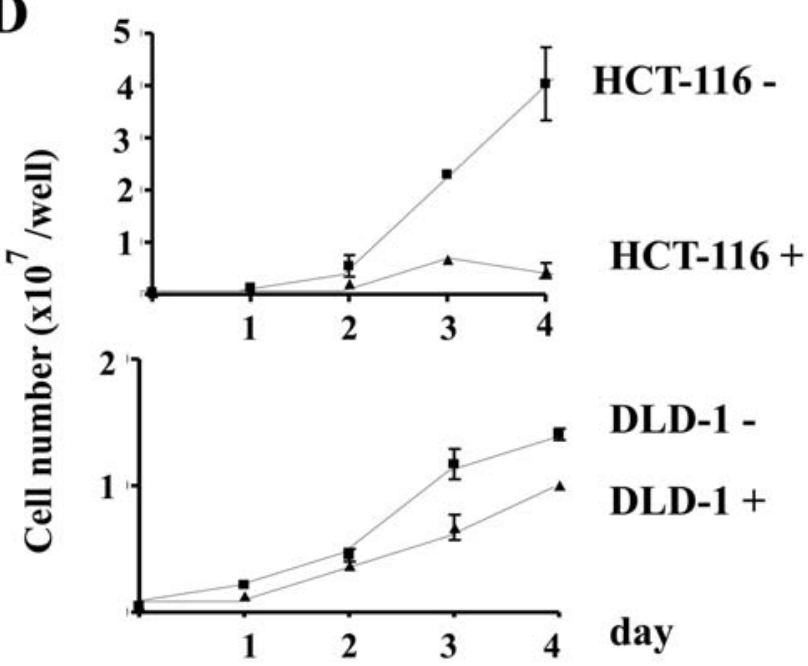

$\mathbf{E}$

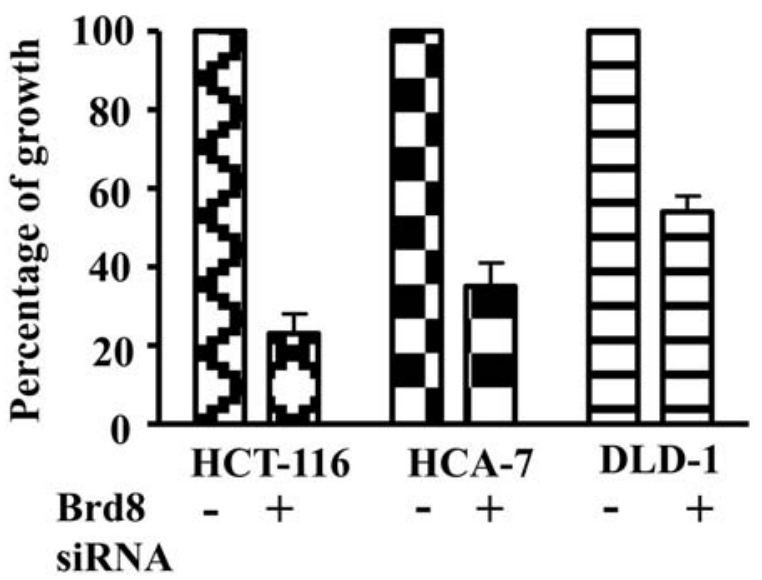

Figure 2. BRD8 knockdown results in growth inhibition. (A) BRD8 knockdown confirmation. We obtained six siRNA constructs for BRD8 (01, 03, 05, s1, s2 and s3; lanes 4-9) along with two negative controls [lane 1, empty transfection; lane 2, green fluorescent protein (GFP)-specific siRNA transfection], and transfected HCT-116 to test protein expression knockdown with immunoblotting. Lane 3 represents the result of a mixture of three siRNA oligo pairs $(01,03$ and 05$)$. Samples were collected $48 \mathrm{~h}$ after transfection, and equal amount of cell extract was loaded to each lane. BRD8 protein amount was decreased to undetectable level in lane 3 and 4. Histone H3 blot is shown for loading control. (B) BRD8 knockdown confirmation in HCT-116, HCA-7 and DLD-1. BRD8 protein expression was suppressed in these cells $48 \mathrm{~h}$ after siRNA transfection. (C) Colony formation assay with BRD8 knockdown. We transfected a set of cultured cells with siRNA (upper row, control; lower row, BRD8 siRNA), incubated them for 96 h, and fixed/stained the cells with 0.5\% methylene blue in $50 \%$ ethanol. In SW-480, HCT-116 and HCA-7, over $60 \%$ of growth inhibition was observed ('sensitive cells'). In HT-29 and DLD-1, growth inhibition was less evident ('less sensitive cells'). (D) Growth curve of HCT-116 (sensitive cells) and DLD-1 (less sensitive cells) with BRD8 inhibition. HCT-116 or DLD-1 cells were seeded $20 \times 10^{4}$ cells/well in 12 well-plate, and transfected with (+) or without (-) BRD8 siRNA at the seeding. Cells were trypsinized and cell number was counted at the indicated time. Presence of BRD8 siRNA (+) inhibited cell proliferation of HCT-116. In DLD-1, cell proliferation continued albeit with slower pace. (E) We quantified cell growth by analyzing cell-staining signals from experiments in (B). Among three cell lines quantified (HCT-116, HCA-7 and DLD-1), HCT-116 and HCA-7 showed significant growth reduction compared with control, but DLD-1 showed less reduction. Experiments were repeated at least three times.

transfection. An equal amount of mixture of 01, 03 and 05 ('mix') showed over 95\% inhibition. Therefore we performed subsequent experiments with the 'mix' (mixture of three
siRNA constructs 01,03 and 05 ) that consistently showed the highest efficiency in knockdown. Independent time-course experiments indicated that the inhibition effect was evident 
A
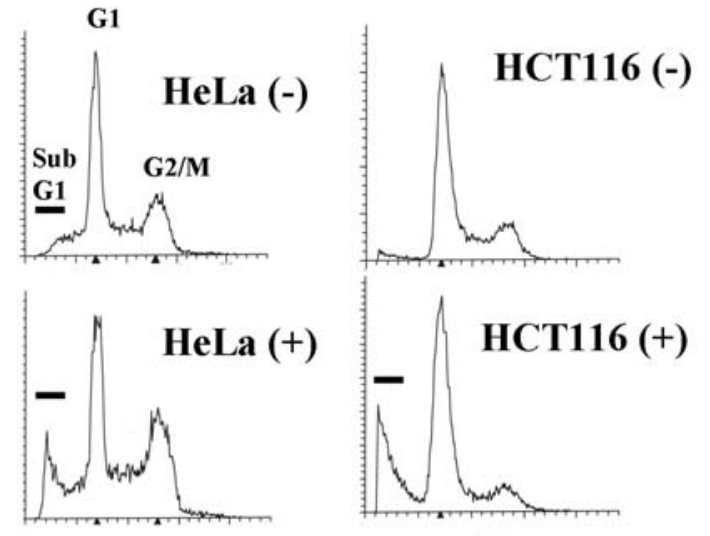
"Sensitive" cell lines
$48 \mathrm{~h}$

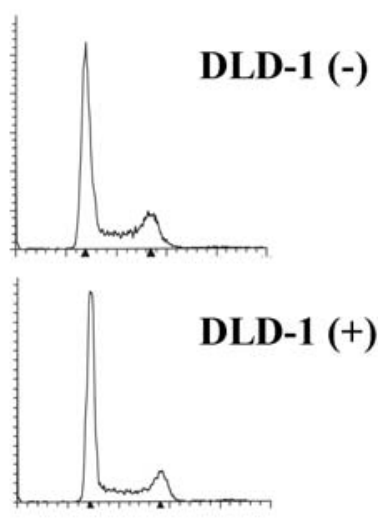

\section{Less sensitive cell line}

$48 \mathrm{~h}$

B

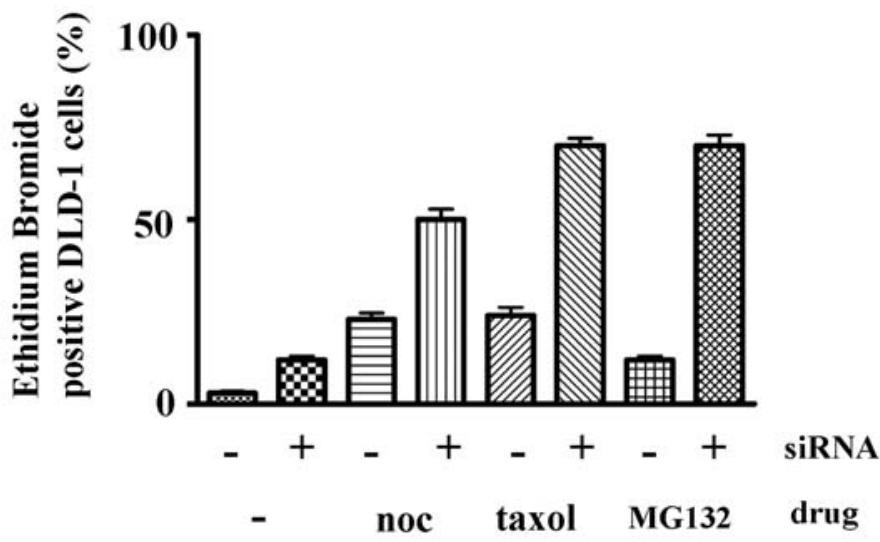

Figure 3. BRD8 knockdown resulted in cell death or chemosensitization. (A) Acute response to BRD8 knockdown varies among cells. BRD8 siRNA transfection to HeLa or HCT-116 cells resulted in an increase in cell death (indicated by subG1 DNA peak corresponding to DNA fragmentation); to DLD-1 resulted in minor increase in G1 cells but acute DNA fragmentation was not evident. (B) Chemosensitization of DLD-1 with BRD8 knockdown. We transfected DLD-1 cells with control or BRD8 siRNA. Forty-eight hours later we treated cells with indicated drug (noc, nocodazole $100 \mathrm{ng} / \mathrm{ml}$; taxol $100 \mathrm{nM}$; MG132 $10 \mu \mathrm{M}$ ) for additional $16 \mathrm{~h}$, and stained cells with acrydine orange/ethidium bromide. Ethidium bromide positive represents cells with compromised membrane integrity and in the process to cell death. Percentage of the ethidium bromide-positive cells is presented in bar graph. BRD8 knockdown cells yielded statistically significant $(\mathrm{P}<0.002)$ difference in dead cell count with drug treatment compared with controls.

$24 \mathrm{~h}$ after transfection, and the inhibition lasted at least for $72 \mathrm{~h}$ (not shown). Other cell lines also showed knockdown, and results for HCT-116, HCA-7 and DLD-1 are shown in Fig. 2B.

We next performed colony formation assays with siRNA transfection using HeLa, SW-480, HCA-7, HT-29, HCT-116, BxPC-3, PANC-1, Caco-2, SkBr-3 and DLD-1. In spite of the difference in basal BRD8 expression level (Fig. 1B), in all cell lines, reduction of colony formation was observed. However, the growth inhibition was more evident (less than $60 \%$ growth compared with control) in SW-480, HCA-7 and HCT-116, and less evident in DLD-1 or HT-29 (Fig. 2C). Fig. 2D presents growth curve of HCT-116 and DLD-1 after transfection with (+) or without (-) BRD8 siRNA, measured with cell number. As shown in Fig. 2C, inhibition of proliferation was more evident in HCT-116 than in DLD-1. Fig. 2E presents quantified results of colony formation of HCT-116, HCA-7 and DLD-1, $96 \mathrm{~h}$ after siRNA transfection compared with control. Growth of BRD8 knocked down HCT-116 averaged $23 \%$ of control; HCA-7 averaged $31 \%$; DLD-1 averaged 58\%. Thus, BRD8 inhibition affected cultured cell growth to a varying degree.
siRNA-mediated BRD8 knockdown resulted in an increase in cell death in 'sensitive' cell lines (e.g., HeLa and HCT-116). We investigated the difference between sensitive cells (e.g., HeLa and HCT-116) and less sensitive cells (e.g., DLD-1). In HeLa and HCT-116 cells, BRD8 siRNA transfection induced an increase in cell population with subG1 DNA content, indicating ongoing DNA fragmentation and cell death (Fig. $3 \mathrm{~A})$. We interpret the increase of cell death as a main cause of significant growth inhibition. In contrast, less sensitive DLD-1 cells show little increase in subG1 population, and only minor increase in $\mathrm{G} 1$ cell population $(\mathrm{G} 1: 55 \%$ in control transfection to $60 \%$ in Brd8 siRNA transfection). The G1 delay may be responsible for the slower growth in DLD-1 cells. We concluded that a major reason for lethality of BRD8 siRNA transfection to sensitive cell lines is induction of cell death.

BRD8 knockdown resulted in specific sensitivity to nocodazole and MG132 in less sensitive DLD-1. BRD8 was isolated as a candidate for spindle poison sensitivity influencing factor in our original screen. We tested whether 

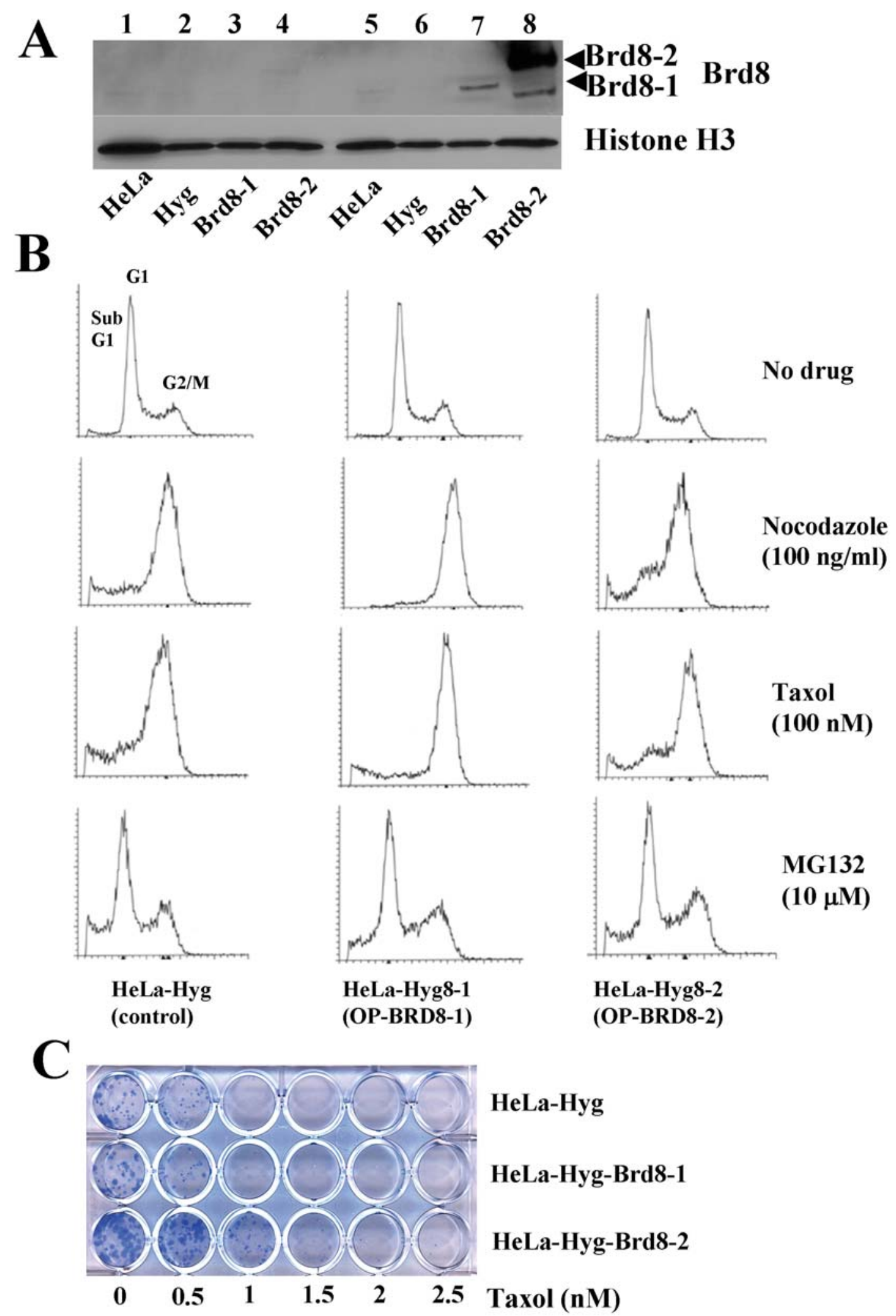

Figure 4. BRD8 overexpression in HeLa cells showed limited protective effect. (A) Establishment of BRD8-overexpressing HeLa cells. We introduced BRD8 expression constructs in HeLa cells and established integrants HeLa-Hyg-BRD8-1 (overexpressing BRD8 isoform 1) and HeLa-Hyg-BRD8-2 (overexpressing BRD8 isoform 2), along with control HeLa-Hyg. To confirm BRD8 expression we performed immunoblotting. BRD8 protein was detected with anti-BRD8 antibody. Lanes 1 and 5, parental HeLa cells; lanes 2 and 6, HeLa-Hyg (control); lanes 3 and 7, HeLa-Hyg-BRD8-1; and lanes 4 and 8, HeLaHyg-BRD8-2. In these cells BRD8 protein amount in normal culture condition was low (left panel, lanes 1-4). However, treatment with a proteasome inhibitor MG132 (10 $\mu \mathrm{M}, 16 \mathrm{~h}$; right panel, lanes 5-8) allowed BRD8 protein to accumulate to a higher level (lanes 7 and 8). Histone $\mathrm{H} 3$ blot is provided for loading control. (B) FACS profile of BRD8 overexpressing cell lines HeLa-Hyg 8-1 (middle column) and HeLa-Hyg-8-2 (right column) along with control HeLa-Hyg (left column), treated with indicated drugs for $16 \mathrm{~h}$. Cell cycle distribution was assessed with Modfit software and the results are shown in Table I. BRD8 overexpressing cells showed a decrease in cells with subG1 DNA content (cell death), indicating an increased short-term resistance to the drugs. (C) BRD8-2 overexpressing cells have growth advantage and show taxol resistance. We seeded HeLa-Hyg, HeLa-Hyg-BRD8-1 and HeLa-Hyg-BRD8-2 (300 cells/well) and treated them with different concentration of taxol $(0,0.5,1,1.5,2$ and $2.5 \mathrm{nM})$. Cells were fixed and stained 8 days after seeding. 
Table I. FACS analysis-cell cycle profiling with drug treatment ( $\%$ of total population).

\begin{tabular}{|c|c|c|c|c|}
\hline & $\begin{array}{l}\text { HeLa-Hyg } \\
\text { (control) }\end{array}$ & HeLa-Hyg BRD8-1 & HeLa-Hyg BRD8-2 & \\
\hline G1 & $50 \%$ & 50 & 50 & No drug \\
\hline $\mathrm{S}$ & 23 & 25 & 23 & \\
\hline $\mathrm{G} 2 / \mathrm{M}$ & 26 & 24 & 27 & \\
\hline subG1 & $<2$ & $<1$ & 2 & \\
\hline G1 & 8 & 2 & 20 & Nocodazole \\
\hline $\mathrm{S}$ & 10 & 6 & 15 & $(100 \mathrm{ng} / \mathrm{ml})$ \\
\hline $\mathrm{G} 2 / \mathrm{M}$ & 72 & 91 & 60 & \\
\hline subG1 & 10 & $<1$ & 5 & \\
\hline G1 & 8 & 4 & 15 & Taxol \\
\hline $\mathrm{S}$ & 11 & 10 & 7 & $(100 \mathrm{nM})$ \\
\hline $\mathrm{G} 2 / \mathrm{M}$ & 61 & 76 & 70 & \\
\hline subG1 & 13 & 10 & 8 & \\
\hline G1 & 38 & 39 & 37 & MG132 \\
\hline $\mathrm{S}$ & 15 & 16 & 18 & $(10 \mu \mathrm{M})$ \\
\hline $\mathrm{G} 2 / \mathrm{M}$ & 29 & 34 & 33 & \\
\hline subG1 & 18 & 11 & 12 & \\
\hline
\end{tabular}

FACS data in Fig. 4B were analyzed with Modfit software to assess cell cycle distribution.

siRNA-mediated BRD8 knockdown affects spindle poison and/or other drug sensitivity in the less sensitive cell line DLD-1. DLD-1 is a spindle poison-resistant colorectal cancer cell line. To observe the effect of BRD8 knockdown in DLD1 on drug treatments under a microscope, we transfected DLD-1 with BRD8 siRNA along with control, and $48 \mathrm{~h}$ later treated cells with spindle poisons nocodazole $(100 \mathrm{ng} / \mathrm{ml})$ or taxol $(100 \mathrm{nM})$ or a proteasome inhibitor MG132 $(10 \mu \mathrm{M})$ for additional $16 \mathrm{~h}$, then stained cells with acrydine orange and ethidium bromide. Red (ethidium bromide positive) stain represents dead/dying cells with compromised membrane integrity. In the presence of spindle poisons nocodazole or taxol, and proteasome inhibitor MG132, BRD8 siRNAtreated cells showed significant increase in ethidium bromide positive cell count (Fig. 3B). The results demonstrated that BRD8 knockdown has cell death-inducing, or chemosensitizing effect to spindle poisons and proteasome inhibitor. The degree of the increase in dead cells (Fig. 3B) suggested that the effect of the drug and BRD8 knockdown may work synergistically, rather than additionally. BRD8 siRNA treatment (Fig. 3B) resulted in an increase in ethidium bromide-positive cells ( $12 \%$ compared with $3 \%$ in control) in DLD-1. We interpret this apparent discrepancy with the FACS result in Fig. 3A as a timing issue. DNA fragmentation detected by FACS analysis was likely preceded by compromise in membrane integrity detected by acridine orange/ethidium bromide staining. Alternatively, BRD8 knockdown may have affected the membrane integrity and changed drug permeability.
Overexpression of BRD 8 in HeLa cells. An increased expression of BRD8 in aggressive colorectal tumors in rat suggested that overexpression of BRD8 may play a role in advanced stage cancers, such as growth advantage or drug resistance. To test this possibility, we established HeLa cellbased BRD8 stably overexpressing cell lines. We reasoned that the effect of overexpression would be more visible in cells whose basal expression of BRD8 is lower, such as HeLa cells, compared with advanced colorectal cancer cell lines (Fig. 1B). We integrated BRD8 expression constructs pCAGGS-BRD8-1-HA (expressing shorter isoform 1) and pCAGGS-BRD8-2-HA (expressing longer isoform 2) in HeLa cell genome along with co-transfected hygromycin resistance gene, and selected hygromycin resistant cells. Unexpectedly, BRD8 protein expression in integrants of BRD8 expression constructs was no higher than controls in exponentially growing culture. However, when cells were treated with the proteasome inhibitor MG132, BRD8 protein showed notable accumulation (Fig. 4A, lanes 7 and 8), indicating that a proteasome-dependent proteolysis system is at least in part involved in downregulation of BRD8 protein in HeLa cells.

We further characterized the BRD8 integrants with FACS analysis (Fig. 4B and Table I). We observed no noticeable difference in the cell cycle profile in exponentially growing culture (Fig. 4B, top row). However, with nocodazole treatment, there was a decrease in subG1 population in BRD8 overexpressing cell lines HeLa-Hyg8-1 and HeLa-Hyg8-2, compared with control HeLa-Hyg. The same decrease in subG1 population was observed with taxol and MG132 
treatment. Thus overexpression of BRD8 altered acute response of cells to spindle poisons or to MG132, and had apparent protective effect.

Finally, we used the cell lines for colony formation assays with nocodazole, taxol and MG132. When seeded the same number of cells, BRD8-2 overexpressing cells formed bigger colonies and were able to grow in taxol concentrations (e.g., $1.5 \mathrm{nM}$ ) in which controls and BRD8-1 overexpressing cells were not (Fig. 4C). This result suggests BRD8-2 overexpression provides growth advantage and taxol resistance. However, from this experiment we were unable to determine whether BRD8-1 provides growth advantage, or the cell line expresses insufficient amount of BRD8-1 to provide significant growth advantage and drug resistance. Also, we were unable to observe significant growth difference in colony formation assays with nocodazole and MG132 (not shown). The drugs may have different or additional effects in longer timespan. Alternatively, the proteasome-dependent BRD8 downregulation mechanism in HeLa cells may be preventing BRD8 from functioning efficiently.

\section{Discussion}

Although this report is the first characterization of BRD8 in cancer cells, there have been three lines of research that suggest BRD8 function. Firstly, BRD8 is one of the bromodomaincontaining proteins. Bromodomain is an acetylated lysinebinding domain (20) and thought to be involved in regulation of protein acetylation and/or HAT (histone acetyl transferase) activity. Secondly, in agreement with the bromodomain function, BRD8 is reported to be an accessory subunit of NuA4 HAT complex (also known as TRRAP/TIP60 complex) through biochemical purification $(17,18)$. Recent research indicated that haplo-insufficiency of TIP60, another component of the NuA4-HAT complex, resulted in increased incidence of tumors in mice and that TIP60 is frequently lost in human tumors, indicating TIP60 acts as a tumor suppressor (21). Thus, one (or more) of HAT complexes containing TIP60 is involved in cell and tumor growth regulation. Thirdly, BRD8 can physically bind to thyroid hormone receptor- $\beta$ and/or retinoid $X$ receptor- $\alpha$, and can act as a coactivator of the nuclear hormone receptor-mediated transcription in reporter assays $(22,23)$. BRD8 was first isolated as a protein that binds to thyroid hormone receptor ligand-binding domain through yeast two-hybrid assay (22). Thus, BRD8 is a part of a signaling pathway that initiates from thyroid hormone or retinoid $\mathrm{X}$. With hormone binding, the hormone receptors interact with other factors including BRD8, and the BRD8containing complex is recruited to and activate core NuA4HAT complex that regulates chromatin remodeling and transcription. Yeast has a counterpart HAT complex also called NuA4-HAT complex $(24,25)$. The core components are conserved between yeast and human, except that human NuA4-HAT complex has two accessory subunits BRD8 and RuvBL1 helicase that are apparently unique to higher eukaryotes including human $(17,18)$. Yeast mutants of the NuA4-HAT complex, e.g., yaf9 $\Delta$, are viable, but show elevated sensitivity to spindle poisons (25). This suggests that the NuA4-HAT complex plays a conserved role in spindle poison response. Given BRD8 is able to interact with the nuclear hormone receptor, we suspect that human BRD8 may be an evolutionary addition to accommodate hormonemediated signaling pathways to the functions of archetypical $\mathrm{NuA} 4$ core complex that include spindle poison response and growth control. A synthetic retinoid and retinoid $\mathrm{X}$ receptor antagonist bexarotene/targretin sensitizes non-small cell lung cancer cells and breast carcinoma to taxol $(26,27)$, implying influence of retinoid pathway on taxol sensitivity in certain cancers.

As shown with TIP60 research, core NuA4-HAT complex is involved in other functions such as regulation of differentiation and maintenance of stem cell identity (28). It may be desirable for therapeutic purpose to specifically target BRD8 and the signaling pathway than to target core NuA4-HAT complex components.

How does BRD8 inhibition induce cell death or sensitize cells to spindle poisons? Given BRD8 is a part of the HAT complex that affects chromatin remodeling and transcription of a set of genes, misregulation of transcription may be a plausible possibility. Such transcriptional change may affect expression of other cell cycle and/or drug resistance genes that may also affect membrane permeability and drug intake, thus BRD8 inhibition acts indirectly. Another possibility is that the loss of a chromatin-interacting protein (BRD8) leads to a change in chromosome structure, perhaps in a critical structure such as kinetochore, and the structural change in chromosomes results in cells prone to mitotic catastrophe caused by spindle poisons.

In summary, we discovered that BRD8 is accumulated in metastatic colon cancer cell lines and advanced rat colorectal cancers. Inhibition of BRD8 via siRNA resulted in induction of cell death or delaying growth. Inhibition of BRD8 also increased the effect of spindle poisons and the proteasome inhibitor. BRD8, and the interacting proteins including NuA4-HAT complex, thyroid hormone receptor and retinoid $\mathrm{X}$ receptor, may define a novel signaling network involved in colorectal tumor cell survival and drug resistance. We propose that targeting BRD8 with secondary drug or siRNA in combination with spindle poison- or proteasome inhibitor would improve chemotherapy against aggressive colorectal cancers, and translational research should follow.

\section{Acknowledgements}

We thank Dr K. Yamaguchi and Dr Y. Furukawa (University of Tokyo, Japan) for sharing unpublished manuscript and for providing BRD8 overexpression constructs. We thank Ms. Alyson Atchison for help in manuscript preparation. This work was supported in part by DOD Breast Cancer Research Grant (DAMD17-02-1-0532) and Kerley-Cade Endowment for Cancer Research (OUHSC).

\section{References}

1. Blagosklonny MV and Fojo T: Molecular effects of paclitaxel: myths and reality (a critical review). Int J Cancer 83: 151-156, 1999.

2. Jordan MA and Wilson L: Microtubules as a target for anticancer drugs. Nat Rev Cancer 4: 253-265, 2004.

3. Jordan MA and Kamath K: How do microtubule-targeted drugs work? An overview. Curr Cancer Drug Targets 7: 730-742, 2007.

4. May KM and Hardwick KG: The spindle checkpoint. J Cell Sci 119: 4139-4142, 2006 
5. Musacchio A and Salmon ED: The spindle-assembly checkpoint in space and time. Nat Rev Mol Cell Biol 8: 379-393, 2007.

6. Weaver BA and Cleveland DW: Decoding the links between mitosis, cancer, and chemotherapy: the mitotic checkpoint, adaptation, and cell death. Cancer Cell 8: 7-12, 2005.

7. Yamada HY and Gorbsky GJ: Spindle checkpoint function and cellular sensitivity to antimitotic drugs. Mol Cancer Ther 5: 2963-2969, 2006.

8. Gascoigne KE and Taylor SS: Cancer cells display profound intra- and interline variation following prolonged exposure to antimitotic drugs. Cancer Cell 14: 111-122, 2008

9. Shi J, Orth JD and Mitchison T: Cell type variation in responses to antimitotic drugs that target microtubules and kinesin-5. Cancer Res 68: 3269-3276, 2008.

10. Whitehurst AW, Bodemann BO, Cardenas J, et al: Synthetic lethal screen identification of chemosensitizer loci in cancer cells. Nature 446: 815-819, 2007.

11. Swanton C, Marani M, Pardo O, et al: Regulators of mitotic arrest and ceramide metabolism are determinants of sensitivity to paclitaxel and other chemotherapeutic drugs. Cancer Cell 11: 498-512, 2007

12. Swanton C, Tomlinson I and Downward J: Chromosomal instability, colorectal cancer and taxane resistance. Cell Cycle 5: 818-823, 2006

13. Mackay H, Hedley D, Major P, et al: A phase II trial with pharmacodynamic endpoints of the proteasome inhibitor bortezomib in patients with metastatic colorectal cancer. Clin Cancer Res 11: 5526-5533, 2005.

14. Yamada HY and Gorbsky GJ: Cell-based expression cloning for identification of polypeptides that hypersensitize mammalian cells to mitotic arrest. Biol Proced Online 8: 36-43, 2006.

15. Yamada HY and Gorbsky GJ: Inhibition of TRIP1/S8/hSug1, a component of the human 19S proteasome, enhances mitotic apoptosis induced by spindle poisons. Mol Cancer Ther 5: 29-38, 2006

16. Ribble D, Goldstein NB, Norris DA and Shellman YG: A simple technique for quantifying apoptosis in 96-well plates. BMC Biotechnol 5: 12, 2005.

17. Cai Y, Jin J, Tomomori-Sato C, et al: Identification of new subunits of the multiprotein mammalian TRRAP/TIP60 containing histone acetyltransferase complex. J Biol Chem 278 : 42733-42736, 2003
18. Doyon Y, Selleck W, Lane WS, Tan S and Côté J: Structural and functional conservation of the NuA4 histone acetyltransferase complex from yeast to humans. Mol Cell Biol 24: 1884-1896, 2004.

19. Tanaka T, Kohno H, Suzuki R, et al: Dextran sodium sulfate strongly promotes colorectal carcinogenesis in Apc (Min/+) mice: inflammatory stimuli by dextran sodium sulfate results in development of multiple colonic neoplasms. Int J Cancer 118: 25-34, 2006.

20. Mujtaba S, Zeng L and Zhou MM: Structure and acetyl-lysine recognition of the bromodomain. Oncogene 26: 5521-5527, 2007.

21. Gorrini C, Squatrito M, Luise C, et al: Tip60 is a haplo-insufficient tumour suppressor required for an oncogene-induced DNA damage response. Nature 448:1063-1067, 2007.

22. Monden T, Wondisford FE and Hollenberg AN: Isolation and characterization of a novel ligand-dependent thyroid hormone receptor-coactivating protein. J Biol Chem 272: 29834-29841, 1997.

23. Monden T, Kishi M, Hosoya T, et al: p120 acts as a specific coactivator for 9-cis-retinoic acid receptor (RXR) on peroxisome proliferator-activated receptor-gamma/RXR heterodimers. Mol Endocrinol 13: 1695-1703, 1999.

24. Allard S, Utley RT, Savard J, et al: $\mathrm{NuA} 4$, an essential transcription adaptor/histone $\mathrm{H} 4$ acetyltransferase complex containing Esa1p and the ATM-related cofactor Tra1p. EMBO J 18: 5108-5119, 1999

25. Le Masson I, Yu DY, Jensen K, et al: Yaf9, a novel NuA4 histone acetyltransferase subunit, is required for the cellular response to spindle stress in yeast. Mol Cell Biol 23: 6086-6102, 2003.

26. Yen WC, Corpuz MR, Prudente RY, et al: A selective retinoid X receptor agonist bexarotene (Targretin) prevents and overcomes acquired paclitaxel (Taxol) resistance in human non-small cell lung cancer. Clin Cancer Res 10: 8656-8664, 2004.

27. Yen WC and Lamph WW: The selective retinoid X receptor agonist bexarotene (LGD1069, Targretin) prevents and overcomes multidrug resistance in advanced breast carcinoma. Mol Cancer Ther 4: 824-834, 2005.

28. Fazzio TG, Huff JT and Panning B: An RNAi screen of chromatin proteins identifies Tip60-p400 as a regulator of embryonic stem cell identity. Cell 134: 162-174, 2008. 